\title{
Направленные поверхностные акустические волны в нецентросимметричных решетках
}

\author{
(C) А.Н. Поддубный \\ Физико-технический институт им. А.Ф.Иоффе Российской академии наук, \\ 194021 Санкт-Петербург, Россия \\ E-mail: poddubny@coherent.ioffe.ru
}

Поступила в Редакцию 30 ноября 2020 г.

В окончательной редакции 7 декабря 2020 г.

Принята к публикации 7 декабря 2020 г.

\begin{abstract}
Рассчитано пространственное распределение поверхностной рэлеевской акустической волны распространяющейся вдоль поверхности полупроводника GaAs, покрытого периодической решеткой параллельных золотых полосок. Показано, что в случае когда решетка не обладает центром пространственной инверсии, распределение деформации для поверхностной волны с блоховским волновым вектором $k_{x}=0$ является асимметричным и характеризуется ненулевым среднем импульсом в плоскости интерфейса и ненулевой степенью циркулярной поляризации в плоскости, перпендикулярной поверхности.
\end{abstract}

Ключевые слова: поверхностные акустические волны, спин, храповики.

DOI: 10.21883/FTP.2021.04.50730.9563

\section{1. Введение}

Управление возбуждениями в полупроводниковых наноструктурах с помощью поверхностных акустических волн является активно использующимся подходом современной физики твердого тела [1]. Так, в работе [2] исследовалась бозе-конденсация экситонных поляритонов в сверхрешетках, индуцированных поверхностной акустической волной. В недавней работе [3] поверхностные акустические волны были использованы для управления двумерным электронным газом, а в работе [4] — для возбуждения квантовых спиновых дефектов.

Важной особенностью поверхностных волн является ненулевая эллиптичность в плоскости, содержащей $k_{x}$ и нормаль к границе $z$ (см. рис. 1) [5]. Действительно, для объемных волн направление вектора деформации $\boldsymbol{u}$ определяется направлением волнового вектора $\boldsymbol{k}$ (вдоль или поперек в зависимости от поляризации). По определению, поверхностная волна затухает от границы, т.е. компонента волнового вектора $k_{z}$, перпендикулярная к границе, имеет ненулевую мнимую часть. Следовательно, компоненты вектора деформации $\boldsymbol{u}$, связанные с комплексным волновым вектором $\boldsymbol{k}=k_{x} \boldsymbol{e}_{x}+k_{z} \boldsymbol{e}_{z}$, $\operatorname{Im} k_{z} \neq 0$, будут иметь разную фазу, и поверхностная волна будет эллиптически поляризована в плоскости $x z$. Знак степени циркулярной поляризации волны

$$
\rho_{c}\left(k_{x}\right)=\frac{2 \operatorname{Im} u_{x} u_{z}^{*}}{|u|^{2}}
$$

зависит от направления распространения волны, $\rho_{c}\left(k_{x}\right)=-\rho_{c}\left(k_{x}\right)$, что является универсальным свойством поверхностных волн различной природы. Аналогичное свойство электромагнитных волн, поверхностных плазмон-поляритонов на границе металла и диэлектрика [6] в последнее время принято называть ненулевым „поперечным спином“", который перпендикулярен волновому вектору плазмона $k_{x}$ и нормали структуры, $\boldsymbol{S} \propto k_{x} \boldsymbol{e}_{x} \times \boldsymbol{e}_{z}$. Благодаря ненулевой эллиптичности, распространяющиеся поверхностные волны перспективны для управления спиновыми степенями свободы [7]. Однако для этого волна должна обладать ненулевым волновым вектором в плоскости структуры $k_{x}$. Например, в эксперименте [4], в котором изучалось взаимодействие поверхностной волны со спиновыми резонансами в дефектах в карбиде кремния, использовалась стоячая волна в пьезоэлектрическом резонаторе, так что в среднем волновой вектор $k_{x}$ равнялся нулю. Альтернативным подходом является генерация поверхностных волн при освещении светом металлических решеток, нанесенных на поверхность полупроводника [8] за счет нагрева и металла при поглощении света. Однако для самых простых одномерных периодических решеток АСАС... (А -

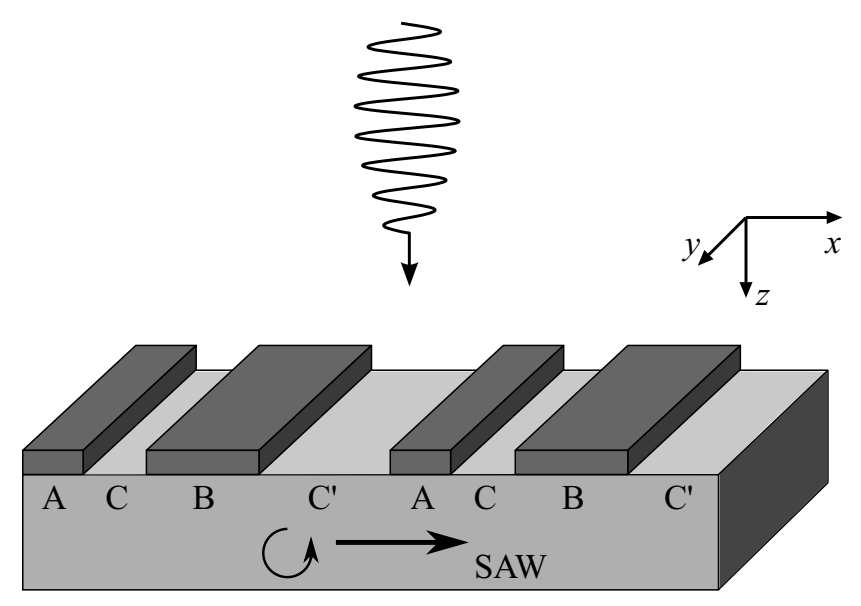

Рис. 1. Схематическое изображение рассматриваемой структуры, состоящей из нецентросимметричной решетки золотых полосок на поверхности GaAs и распространяющейся в ней поверхностной акустической волны. 
металлические полоски, С - щели между ними) есть центр пространственной инверсии. Поэтому в среднем волновой вектор $k_{x}$ для поверхностной волны внутри решетки также равен нулю и средняя циркулярная поляризация отсутствует.

В настоящей работе рассмотрены поверхностные рэлеевские акустические волны, возникающие в полупроводнике, покрытом решеткой полосок $\mathrm{ACBC}^{\prime} \mathrm{ACBC}^{\prime} \ldots$ без центра инверсии (А и В - полоски, $\mathrm{C} \mathrm{C}^{\prime}-$ щели), как показано на рис. 1. Подобные латеральные решетки ранее использовались при изучении храповика Зеебека - ненулевого постоянного тока электронов, возникающего в графене или гетероструктуре, покрытом решеткой при освещении терагерцовым излучением [9-11]. По аналогии с эффектом электронного храповика в работах [9-11] изучаемый эффект направленной генерации акустических волн с ненулевой степенью циркулярной поляризации можно назвать „акустическим спиновым храповиком“.

Работа построена следующим образом. В разд. 2 представлена теоретическая модель и метод расчета. Результаты расчета обсуждаются в разд. 3 и в разд. 4 представлено заключение.

\section{2. Модель и метод расчета}

Рассматриваемая структура схематически показана на рис. 1. Она представляет собой объемный полупроводник GaAs, на поверхность которого нанесена бесконечная периодическая решетка металлических полосок с периодом $d=a+b+c+c^{\prime}$. Акустические волны на частоте $\omega=2 \pi v$ описываются уравнением теории упругости

$$
-\omega^{2} \rho u_{\alpha}=\partial_{\beta} \sigma_{\alpha \beta},
$$

где $\boldsymbol{u}$ - вектор деформации, и $\sigma_{\alpha \beta}=c_{\alpha \beta \gamma \delta} \partial_{\gamma} u_{\delta}-$ тензор напряжений, и $\rho-$ плотность материала, $\alpha, \beta=x, y, z$. Для GaAs использовались следующие значения упругих параметров: $c_{11}=1.18 \cdot 10^{12}$ дин $/ \mathrm{cm}^{2}$, $c_{12}=0.53 \cdot 10^{12}$ дин $/ \mathrm{cm}^{2}, \quad c_{44}=0.59 \cdot 10^{12}$ дин $/ \mathrm{cm}^{2}, \rho=$ $=5.3 г / \mathrm{cm}^{3}$ из работы [12]. На границе GaAs и золотых полосок, следуя работе [13], были использованы упрощенные граничные условия [5], при которых влияние полосок сводится к нагрузке с линейной плотностью массы $\mu(x)$, что описывается уравнением

$$
\omega^{2} \mu(x) u_{\beta}(z=0)=\sigma_{z, \beta}(z=0), \quad \beta=x, y, z .
$$

В данной работе мы ограничимся частным случаем волн, распространяющихся вдоль оси $x \|$ [001] и поляризованных в плоскости $x z$, так что $u_{y}=0, \partial_{y} \boldsymbol{u}=0$. Для решения уравнений (2), (3) был использован стандартный подход, основанный на разложении вектора деформации по плоским волнам,

$$
\boldsymbol{u}(x, z)=\sum_{h=-\infty}^{\infty} e^{\mathrm{i}\left(k_{x}+2 \pi h / d\right) x} \boldsymbol{u}_{h}(z)
$$

и

$$
\mu(x)=\sum_{h=-\infty}^{\infty} e^{2 \pi \mathrm{i} h x / d} \mu_{h}, \quad \mu_{h}=\frac{1}{d} \int_{0}^{d} \mathrm{~d} x \mu(x) e^{-2 \pi \mathrm{i} h x / d},
$$

где $k_{x}$ - блоховский волновой вектор вдоль решетки. Здесь линейная плотность

$$
\mu(x)=\left\{\begin{aligned}
\rho^{\prime} H, & 0<x \leq a, \quad a+c<x \leq a+c+b, \\
0, & a<x<a+c, \quad a+c+b<x \leq d,
\end{aligned}\right.
$$

где $\rho_{c}=19.3$ г $/ \mathrm{cm}^{3}-$ объемная плотность золота и $H-$ толщина полосок. Для каждой из гармоник $\propto e^{\mathrm{i}\left(k_{x}+2 \pi h / d\right) x}$ решение может быть представлено в виде

$$
\boldsymbol{u}_{h}(z)=\sum_{\sigma= \pm} \sum_{\lambda=L, T} A_{\eta} e^{\mathrm{i} k_{z, \eta} z} \boldsymbol{u}_{\eta}
$$

где мультииндекс $\eta \equiv(\sigma, \lambda, h)$, знак $\sigma$ определяет направление распространения волны (в полупроводник или из него) с соответствующим волновым вектором $k_{z}$, а индекс $\lambda$ характеризует поляризацию волны $\boldsymbol{u}_{\eta}$ и $A_{\eta}-$ искомые коэффициенты разложения. Значения волновых векторов $k_{z, \eta}$ и векторов деформации $\boldsymbol{u}_{\eta} \equiv\left[u_{\eta, x}, u_{\eta, z}\right]^{T}$ определяются из независимого решения задачи на собственные значения (2) для каждой из гармоник или в явном виде:

$$
\left(\begin{array}{cc}
c_{11} k_{x, h}^{2}+c_{44} k_{z, \eta}^{2} & \left(c_{12}+c_{44}\right) k_{z, \eta} k_{x, h} \\
\left(c_{12}+c_{44}\right) k_{z, \eta} k_{x, h} & c_{44} k_{x, h}^{2}+c_{11} k_{z, \eta}^{2}
\end{array}\right) \boldsymbol{u}_{\eta}=\omega^{2} \boldsymbol{u}_{\eta},
$$

где $k_{x, h}=k_{x}+2 \pi h / d$.

Подставляя разложения (5), (7) в граничные условия (3) и приравнивая нулю коэффициенты при всех гармониках $\propto e^{\mathrm{i}\left(k_{x}+2 \pi h / d\right) x}$, можно получить систему связанных линейных уравнений на коэффициенты $A_{\eta}$. Для определения дисперсии поверхностных волн из этой системы можно получить замкнутую однородную систему уравнений вида $S_{h \beta ; v^{\prime}} A_{v^{\prime}}=0, \beta=x, z$, оставив в разложении (7) только затухающие в глубь полупроводника гармоники, для которых $\operatorname{Im} k_{z, \lambda h \sigma}>0$. В явном виде матрица $S$ равняется

$$
S_{h \beta ; \nu^{\prime}}=\delta_{h h^{\prime}} \sigma_{z}\left(\left[\boldsymbol{u}_{\eta}\right]\right)_{\beta}-\mu_{h-h^{\prime}} \omega^{2}\left[\boldsymbol{u}_{\eta^{\prime}}\right]_{\beta},
$$

где

$$
\sigma_{z}\left(\left[\boldsymbol{u}_{\eta}\right]\right)=\mathrm{i}\left(\begin{array}{c}
c_{44}\left(k_{x, h} u_{\eta, z}+k_{z, \eta} u_{\eta, x}\right) \\
c_{11} k_{z, \eta} u_{\eta, z}+c_{12} k_{x, h} u_{\eta, x}
\end{array}\right) .
$$

Удерживая конечное число гармоник $h=$ $=-h_{\max } \ldots h_{\max }$, можно получить частоты поверхностных волн из решения уравнения $\operatorname{det}\left|S\left(\omega, k_{x}\right)\right|=0$. Однако частоты поверхностных волн $\omega$ оказываются комплексными из-за гибридизации с объемными решениями, индуцированной дифракционной решеткой волны могут „утекать“ в глубь полупроводника. Решение уравнения $\operatorname{det}\left|S\left(\omega, k_{x}\right)\right|=0$ на комплексные частоты $\omega$ 
является численно трудоемкой задачей. Приближенное значение вещественных частей частот поверхностных волн может быть получено путем минимизации определителя при вещественных значениях $\omega$.

В качества альтернативы задаче на собственные значения может быть решена задача рассеяния. Это аналогично подходу для электромагнитных поверхностных волн - плазмон-поляритонов, см. например, [14,15]. В этом случае считается, что на решетку из глубины полупроводника падает объемная волна

$$
\boldsymbol{u}(\boldsymbol{r})=\boldsymbol{u}_{\lambda_{0}, 0,-} e^{\mathrm{i} k_{x} x-\mathrm{i}\left|k_{z}\right| z}
$$

определенной поляризации $\lambda_{0}$ и рассчитывается деформация, возбуждаемая такой волной во всей структуре. Значения коэффициентов $A_{v}$ определяются неоднородной системой уравнений

$$
S_{h \beta ; v^{\prime}} A_{v^{\prime}}=S_{h \beta ; v^{\prime}} \delta_{h^{\prime} 0} \delta_{\lambda \lambda_{0}} \delta_{\sigma,-} .
$$

Вещественные части комплексных частот квазистационарных поверхностных волн будут определяться положениями резонансов в частотно-зависимых коэффициентах $A_{v}$. В настоящей работе таким образом вычислялся коэффициент зеркального отражения продольной акустической волны $r \equiv A_{0, L,+}$. Как будет показано в следующем разделе, спектральные особенности в коэффициенте отражения соответствуют резонансу с поверхностной акустической волной.

\section{3. Результаты и обсуждение}

На рис. 2 цветом представлена зависимость спектра отражения продольной акустической волны, падающей на структуру, от волнового вектора $k_{x}$ и частоты вол-

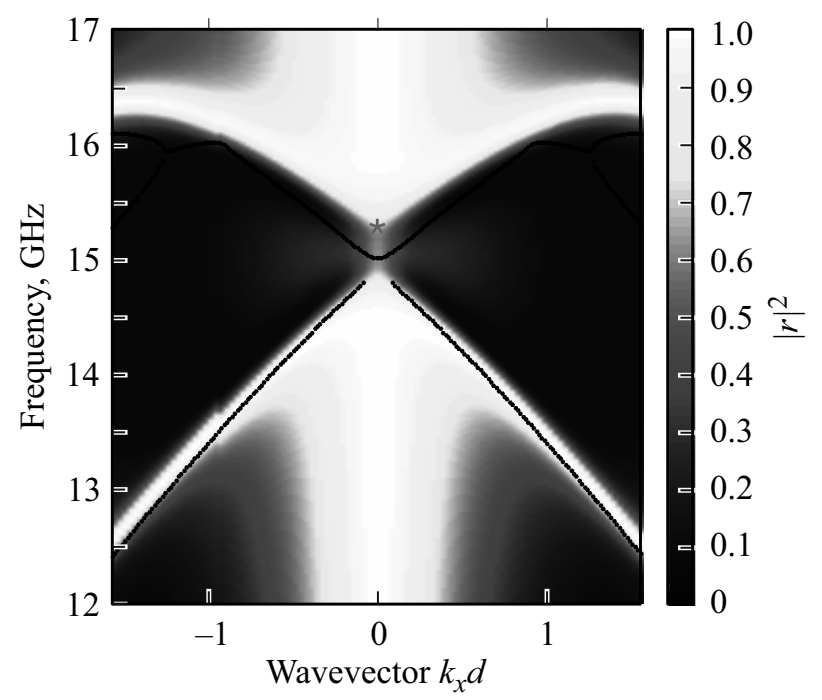

Рис. 2. Зависимость коэффициента отражения падающей продольной акустической волны от частоты $v$ и волнового вектора $k_{x}$. Точками показана рассчитанная вещественная частота собственных волн в структуре. Расчет выполнен для $a=c^{\prime}=20 \mathrm{нм}, \quad b=c=2 a, H=15 \mathrm{HM}, h_{\max }=2$. Значения остальных параметров указаны в тексте.
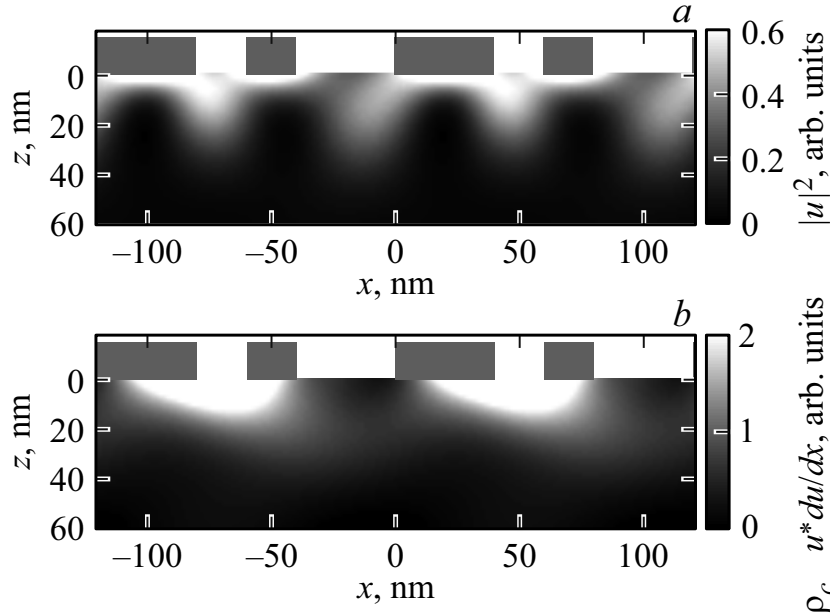

Рис. 3. Карта пространственного распределения интенсивности деформации $\left|\boldsymbol{u}^{2}\right|$ (a) параметра асимметрии $\boldsymbol{u}^{*} \partial_{x} \boldsymbol{u}(b)$ степени локальной циркулярной поляризации $\rho_{c}=2 \operatorname{Im} u_{x} u_{z}^{*} /\left|\boldsymbol{u}^{2}\right| \quad(c)$. Расчет выполнен для $k_{x}=0$ и $v=15.2$ ГГц (что соответствует звездочке на рис. 2). Значения остальных параметров такие же, как и на рис. 2.

ны $v$. Точки соответствуют приближенному расчету вещественных частей поверхностной волны, минимизируя значения определителя $\operatorname{det}\left|S\left(\omega, k_{x}\right)\right|$. Расчет демонстрирует два резонанса, соответствующих „сложенным“ акустическим волнам, с дисперсией $\omega\left(k_{x}\right)=v\left|k_{x} \pm 2 \pi / d\right|$, испытывающим антипересечение в области $k_{x}=0$, где $d=120$ нм - период структуры, и $v \approx 1.8 \mathrm{\kappa м} / \mathrm{c}-$ скорость поверхностных волн. Отметим, что скорость поверхностных волн оказывается меньше, чем значение скорости для рэлеевских волн в объемном $\mathrm{GaAs}$ $v \approx 2.7$ км/с [12], что связано с нагрузкой поверхности золотыми полосками.

Ширина спектрального провала в спектре отражения при $k_{x}=0$ составляет $\sim 0.5$ ГГц и соответствует запрещенной зоне в спектре поверхностных волн. Два подхода к решению, на основе задачи на собственные значения и на основе задачи рассеяния, достаточно хорошо соответствуют друг другу, за исключением узкой области волновых векторов вблизи $k_{x}=0$. В этом случае ширина спектрального резонанса для нижней ветки сложенных поверхностных колебаний становится сопоставима с шириной запрещенной зоны, и различить нижнюю ветку сложенных колебаний на основе минимизации $\operatorname{det}\left|S\left(\omega, k_{x}=0\right)\right|$ при вещественных $\omega$ оказывается затруднительно.

На рис. 3 показано пространственное распределение абсолютного значения вектора деформации 

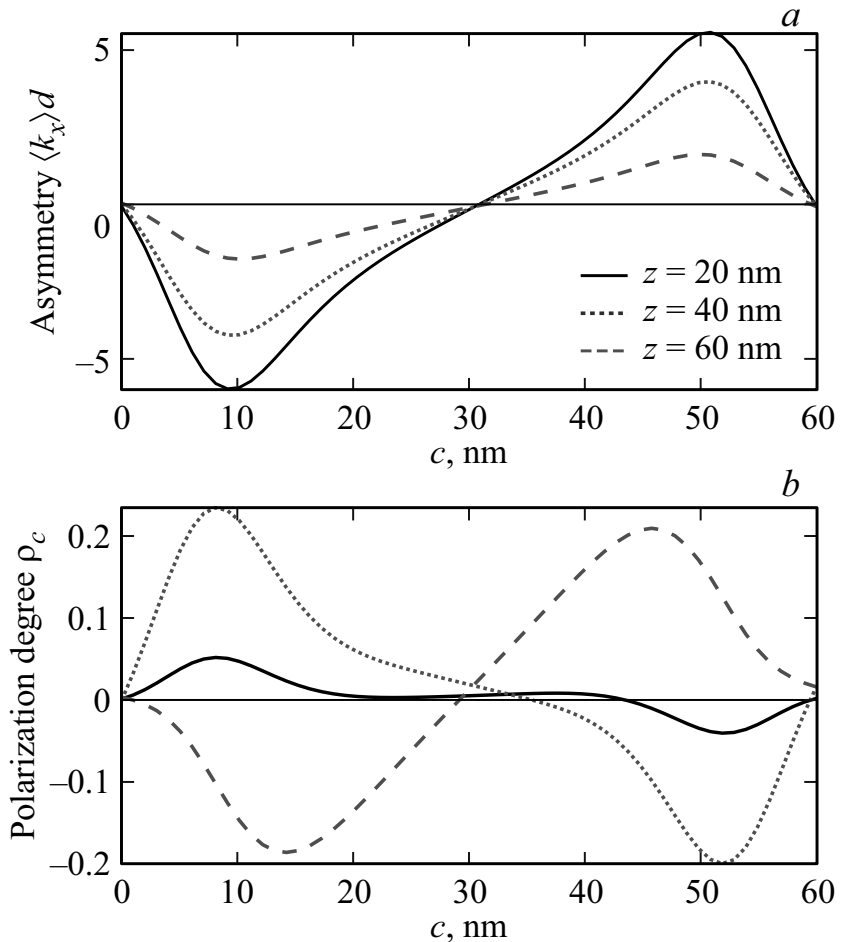

Рис. 4. Зависимость параметра асимметрии $\left\langle k_{x}\right\rangle d \equiv \boldsymbol{u}^{*} \partial_{x} \boldsymbol{u}(a)$ и степени локальной циркулярной поляризации $\rho_{c}=$ $=2 \operatorname{Im} u_{x} u_{z}^{*} /\left|\boldsymbol{u}^{2}\right|(b), \quad$ от расстояния $c$ при постоянном $c+c^{\prime}=60$ нм. Параметры были усреднены по координате $x$ внутри элементарной ячейки. Сплошные, пунктирные и штриховые кривые рассчитаны для глубин $z=20,40,60$ нм. Значения остальных параметров такие же, как и на рис. 2.

$\left|u^{2}\right|=\left|u_{x}\right|^{2}+\left|u_{z}\right|^{2}(a)$, эффективного „волнового вектора“ $^{\text {“c }} \boldsymbol{u}^{*} \cdot \partial_{x} \boldsymbol{u}(b)$ и степени циркулярной поляризации волны (1) (c). Карты рассчитаны при возбуждении структуры по нормали, т.е. $k_{x}=0$ на частоте $v=15.2$ ГГц (что соответствует звездочке на рис. 2). Как и ожидается для поверхностной волны, колебания на рис. $3, a, b$ сосредоточены вблизи границы полупроводника и решетки и быстро спадают при удалении от границы. Распределение вектора деформации оказывается существенно модулированным решеткой и асимметричным в пространстве. Это особенно заметно на рис. 3, $b$, где величина $\boldsymbol{u}^{*} \cdot \partial_{x} \boldsymbol{u}$, определяющая направленность распределения деформации [11], имеет один и тот же знак в пределах элементарной ячейки. Аналогичный расчет, выполненный для пространственно-симметричной ячейки, дает знакопеременный ответ для $\boldsymbol{u}^{*} \cdot \partial_{x} \boldsymbol{u}$, обращающийся в нуль при усреднении по $x$. Значение асимметрии $\boldsymbol{u}^{*} \cdot \partial_{x} \boldsymbol{u}$ максимально в узком слое вблизи поверхности структуры толщиной 10-20 нм, при этом волна сосредоточена под полосками А и В. В этой области степень циркулярной поляризации волны близка к единице (см. рис. 3,c). При удалении от поверхности степень циркулярной поляризации меняет знак. Однако интенсивность деформации также уменьшается, поэтому наибольший „поперечный спин“ $2 \operatorname{Im} u_{x} u_{z}^{*}$ положителен и сосредоточен вблизи поверхности.
На рис. 4 показана зависимость усредненных по элементарной ячейке параметров асимметрии и степеней циркулярной поляризации, рассчитанных в зависимости от толщины слоя $c$ при постоянном $c+c^{\prime}$, т.е. сдвиге полоски В в пределе элементарной ячейки для трех различных глубин в полупроводнике $z=20,40,60$ нм. Максимальная степень асимметрии достигается при $c \approx a / 2 \approx 10$ нм или $c^{\prime} \approx 10$ нм. Усредененная степень циркулярной поляризации $\rho_{c}$ в этом случае также максимальна. Значения $c=0, c^{\prime}=0, c=c^{\prime}$, когда структура оказывается центрально-симметричной, соответствуют $\left\langle\boldsymbol{u}^{*} \cdot \partial_{x} \boldsymbol{u}\right\rangle=0$.

\section{4. Заключение}

В работе произведен расчет распределения ближнего поля поверхностной рэлеевской акустической волны в полупроводнике GaAs, покрытом периодической нецентросимметричной решеткой параллельных золотых полосок. Рассчитана зависимость асимметрии распределения волны и ее средней степени циркулярной поляризации от параметра асимметрии решетки и от расстояния до поверхности. Расчет указывает на возможность генерации поверхностных акустических волн с высокой степенью поперечной циркулярной поляризации $\rho_{c}>0.5$ и частотами $v \geq 10$ ГГц $(\hbar \omega \sim 50$ мк $\cdot \mathrm{eB})$. Такие нецентросимметричные решетки могут быть актуальны для управления спиновыми степенями свободы носителей заряда и экситонов в наноструктурах. В частности, поглощение поверхностных акустических волн может приводить к спин-зависимым переходам между состояниями легких и тяжелых дырок в валентной зоне GaAs [4].

\section{Благодарности}

Автор благодарит И.А. Акимова, В.Л. Коренева, С.М. Кухтарука и А.В. Пошакинского за полезные обсуждения.

\section{Финансирование работы}

Работа выполнена при поддержке гранта РНФ 20-1200194.

\section{Конфликт интересов}

Автор заявляет об отсутствии конфликта интересов.

\section{Список литературы}

[1] M.M. de Lima,jr, P.V. Santos. Rep. Prog. Phys., 68 (7), 1639 (2005).

[2] E.A. Cerda-Méndez, D.N. Krizhanovskii, M.S. Skolnick, P.V. Santos. J. Phys. D, 51 (3), 033001 (2017).

[3] B.Friess, I.A. Dmitriev, V.Umansky, L. Pfeiffer, K. West, K. von Klitzing, J.H. Smet. Phys. Rev. Lett., 124, 117601 (2020). 
[4] A. Hernández-Mínguez, A.V. Poshakinskiy, M. Hollenbach, P.V. Santos, G.V. Astakhov. Phys. Rev. Lett., 125, 107702 (2020).

[5] B.A. Auld. Acoustic fields and waves in solids (N. Y., Wiley and Sons, 1973) v. 1.

[6] K.Y. Bliokh, F.J. Rodríguez-Fortuño, F. Nori, A.V. Zayats. Nature Photonics, 9 (12), 796 (2015).

[7] F. Spitzer, A.N. Poddubny, I.A. Akimov, V.F. Sapega, L. Klompmaker, L.E. Kreilkamp, L.V. Litvin, R. Jede, G. Karczewski, M. Wiater, T. Wojtowicz, D.R. Yakovlev, M. Bayer. Nature Physics, 14 (10), 1043 (2018).

[8] D. Munk, M. Katzman, M. Hen, M. Priel, M. Feldberg, T. Sharabani, Sh. Levy, A. Bergman, A. Zadok. Nature Commun., 10 (1), 4214 (2019).

[9] P. Olbrich, E.L. Ivchenko, R. Ravash, T. Feil, S.D. Danilov, J. Allerdings, D. Weiss, D. Schuh, W. Wegscheider, S.D. Ganichev. Phys. Rev. Lett., 103, 090603 (2009).

[10] A.V. Nalitov, L. E. Golub, E.L. Ivchenko. Phys. Rev. B, 86, 115301 (2012).

[11] Е.Л. Ивченко, М.И. Петров. ФТТ, 56, 1772 (2014).

[12] A.A. Maznev, A.M. Lomonosov, P. Hess, A.A. Kolomenskii. Eur. Phys. J. B, 35 (3), 429 (2003).

[13] A.A. Maznev, A.G. Every. J. Appl. Phys., 106 (11), 113531 (2009).

[14] Н.А. Гиппиус, С.Г. Тиходеев. Успехи физ. наук, 179 (9), 1027 (2009).

[15] V.A. Zakharov, A.N. Poddubny. Phys. Rev. A, 101, 043848 (2020).

Редактор А.Н. Смирнов

\title{
Directional surface acoustic waves in noncentrosymmetric gratings
}

\author{
A.N. Poddubny \\ loffe Institute, \\ 194021 St. Petersburg, Russia
}

Abstract Spatial distribution of surface Rayleigh acoustic wave propagating along the surface of GaAs semiconductor covered by a periodic grating of gold stripes is calculated. We demonstrated that when the lattice has no center of spatial inversion the distribution of deformation for the surface wave with the Bloch wave vector $k_{x}=0$ is asymmetric and characterized by nonzero mean momentum in the interface plane and nonzero degree of transverse polarization in the plane perpendicular to the surface. 